\title{
Preface-S.I.: LATIN 2014
}

\author{
Alfredo Viola 1
}

Received: 21 June 2016 / Accepted: 23 June 2016 / Published online: 14 July 2016

(C) Springer Science+Business Media New York 2016

\section{Preface}

This special issue contains the journal versions of a selection of papers from the 11th Latin American Symposium on Theoretical Informatics (LATIN), held on March 31-April 4 2014, in Montevideo, Uruguay. The conference covered a broad range of topics in theoretical computer science, including algorithms, analytic and enumerative combinatorics, analysis of algorithms, approximation algorithms, automata theory, combinatorics and graph theory, complexity theory, computability, computational algebra, computational geometry, data structures, graph drawing, and random structures. Eight papers were selected for this special issue, all of which went through the thorough reviewing process that is the standard for Algorithmica. The selection was based on two criteria: the quality of the manuscripts and the desire to give readers a sense of the breadth and depth of the topics covered by LATIN. The overview that follows underscores these points

In A Randomized Incremental Algorithm for the Hausdorff Voronoi Diagram of Non-crossing Clusters, Cheilaris, Khramtcova, Langerman and Papadopoulou present a construction based on point location, that computes this diagram in expected $O\left(n \log ^{2} n\right)$ time and expected $O(n)$ space. Their techniques efficiently handle nonstandard characteristics of generalized Voronoi diagrams, such as sites of non-constant complexity, sites that are not enclosed in their Voronoi regions, and empty Voronoi regions.

The conjugacy problem asks whether two words over generators of a fixed group $G$ are conjugated. In Conjugacy in Baumslag's group, generic case complexity,

Alfredo Viola

viola@fing.edu.uy

1 Universidad de la República, Montevideo, Uruguay 
and division in power circuits, Diekert, Myasnikov and Weiß investigate the conjugacy problem for two prominent groups: the Baumslag-Solitar group $\mathrm{BS}_{1,2}$ and the Baumslag group $\mathrm{G}_{1,2}$ (also known as Baumslag-Gersten group). They prove that the conjugacy problem in $\mathrm{BS}_{1,2}$ is complete for the circuit class $\mathrm{TC}^{0}$. Moreover, they show that the conjugacy problem in $\mathrm{G}_{1,2}$ can be solved in polynomial time in a strongly generic setting. In contrast, they prove that under a plausible assumption the average case complexity of the same problem is non-elementary.

The signature of a permutation $\sigma$ is a word $\operatorname{sg}(\sigma) \subseteq\{\mathfrak{a}, \mathfrak{d}\}^{*}$ with $i$ th letter $\mathfrak{d}$ when $\sigma$ has a descent (i.e. $\sigma(i)>\sigma(i+1)$ ) and $\mathfrak{a}$ when $\sigma$ has an ascent (i.e. $\sigma(i)<\sigma(i+$ $1))$. In Counting and generating permutations in regular classes, Basset introduces regular classes of permutations, the sets $\Lambda(L)$ of permutations with signature in regular languages $L \subseteq\{\mathfrak{a}, \mathfrak{d}\}^{*}$. Given a regular class of permutations the paper counts those of a given length within the class, computes a closed form formula for their exponential generating function, and gives an algorithm to uniformly sample at random some permutation of a given length.

The problem 2-Xor-Sat asks for the probability that a random expression, built as a conjunction of clauses $x \oplus y$, is satisfiable. This problem is addressed by de Panafieu, Gardy, Gittenberger and Kuba in 2-Xor revisited: satisfiability and probabilities of functions. They give a new, explicit expression of this probability, and then they study the probability that a random expression computes a specific Boolean function. The analysis involves an interesting description of 2-Xor expressions as multi graphs and use classical methods of analytic combinatorics by expressing probabilities through coefficients of generating functions.

Given $\mathrm{n}$ jobs represented as d-dimensional vectors in $[0,1]^{d}$, and $\mathrm{m}$ identical machines, the goal of the Vector Scheduling problem is to assign the jobs to machines such that the maximum load of each machine over all the coordinates is at most 1. This problem is studied in Approximating Vector Scheduling: almost matching upper and lower bounds by Bansal, Oosterwijk, Vredeveld and van der Zwaan where they show that a double exponential dependence on $d$ is necessary, and give an improved algorithm with essentially optimal running time.

In Biased Predecessor Search, Bose, Fagerberg, Howat and Morin consider the problem of performing predecessor searches in a bounded universe while achieving query times that depend on the distribution of queries. They give data structures that achieve expected query times logarithmic in the entropy of the distribution of queries and with space bounded in terms of universe size. They also present data structures that use only linear space but with query times that are higher (but still sub linear) functions of the entropy. Moreover, they study individual query times on universe elements with general weights, as well as the case when the distribution is not known in advance.

The article Generalized and Quotient Models for Random And/Or Trees and Application to Satisfiability by Genitrini and Mailler is motivated by the following satisfiability question: pick uniformly at random an and/or Boolean expression of length $n$, built on a set of $k_{n}$ Boolean variables. What is the probability that this expression is satisfiable? asymptotically when $n$ tends to infinity? The model of random Boolean expressions developed in the paper is the model of Boolean Catalan trees, already extensively studied in the literature for a constant sequence $\left(k_{n}\right)_{n \geq 1}$. 
The fundamental breakthrough of this paper is to generalize the previous results for any (reasonable) sequence of integers $\left(k_{n}\right)_{n \geq 1}$, which enables us, in particular, to solve the above satisfiability question.

The Connected Facility Location (CFL) is a network design problem that arises from a combination of the Uncapacitated Facility Location (FL) and the Steiner Tree (ST) problems. The Online Connected Facility Location problem (OCFL) is an online version of the CFL. In $A$ Randomized $O(\log n)$-Competitive Algorithm for the Online Connected Facility Location Problem, San Felice, Williamson and Lee analyze an asymptotic optimal $O(\log n)$-competitive randomized algorithm for OCFL.

I thank the authors for accepting my invitation, the referees for their careful reviews, and Ming-Yang Kao - the editor-in-chief of Algorithmica-for giving me the opportunity to edit this special issue. Special thanks go to Rohini Duraisingh for her invaluable help in the refereeing process.

June 15, 2016

La Floresta

Alfredo Viola 